\title{
Early onset of efficacy with fremanezumab in patients with episodic and chronic migraine: subanalysis of two phase $2 \mathrm{~b} / 3$ trials in Japanese and Korean patients
}

Takao Takeshima ${ }^{1}$, Masami Nakai ${ }^{2}$, Yoshiyuki Shibasaki ${ }^{3}$, Miki Ishida ${ }^{4}$, Byung-Kun Kim $^{5}$, Xiaoping Ning ${ }^{6}$ and Nobuyuki Koga ${ }^{7}$

\begin{abstract}
Background: Early onset of action has become recognized as an important efficacy feature of preventive migraine treatment, which can help overcome adherence issues commonly associated with older medications. Preventive treatments that target the calcitonin gene-related peptide (CGRP) or the CGRP receptor have been previously shown to provide early onset of action.

Methods: This subanalysis of primary endpoints of two separate phase $2 \mathrm{~b} / 3$ studies sought to determine the onset of action of fremanezumab in Japanese and Korean patients with episodic migraine (EM) and chronic migraine (CM).

Results: In EM patients ( $n=357$ ), both fremanezumab quarterly and fremanezumab monthly led to greater reductions in weekly migraine days (days/week) than placebo from the first week after the initial injection and thereafter during the remainder of the study period. Similarly, CM patients $(n=571)$ had a greater reduction in headache days of at least moderate severity (days/week) with fremanezumab (total) than placebo. The percentage of patients with a migraine day (EM) or headache day at least moderate severity (CM) was lower in those treated with fremanezumab than placebo and this effect was apparent from as early as Day 2 (1 day after first injection).
\end{abstract}

Conclusions: These results suggest that fremanezumab has an early onset of action, as noted in previous post hoc analyses of anti-CGRP monoclonal antibodies.

Trial registration: ClinicalTrials.gov. NCT03303092, Registered 5 October 2017, NCT03303079, Registered 5 October 2017.

Keywords: Calcitonin gene-related peptide, Chronic migraine, Episodic migraine, Fremanezumab, Japanese, Korean, Early onset

*Correspondence: Nakai.Masami@otsuka.jp

2 Medical Affairs, Otsuka Pharmaceutical Co., Ltd., 3-2-27 Otedori, Chuo-ku, Osaka 540-0021, Japan

Full list of author information is available at the end of the article

\section{Introduction}

Both chronic migraine (CM) and episodic migraine (EM) are associated with significant pain and disability as well as impairments in quality of life, functioning, and interpersonal relationships [1-6]. Existing oral preventive treatments are limited by suboptimal efficacy, adverse events, and poor adherence [7-12]. Issues with adherence relate to both tolerability and poor efficacy, which permits use, sharing, adaptation, distribution and reproduction in any medium or format, as long as you give appropriate credit to the original author(s) and the source, provide a link to the Creative Commons licence, and indicate if changes were made. The images or other third party material in this article are included in the article's Creative Commons licence, unless indicated otherwise in a credit line to the material. If material is not included in the article's Creative Commons licence and your intended use is not permitted by statutory regulation or exceeds the permitted use, you will need to obtain permission directly from the copyright holder. To view a copy of this licence, visit http://creativecommons.org/licenses/by/4.0/. The Creative Commons Public Domain Dedication waiver (http://creativeco mmons.org/publicdomain/zero/1.0/) applies to the data made available in this article, unless otherwise stated in a credit line to the data. 
in turn is associated with delays in gaining maximum effect during dose titration $[13,14]$. One result of this is a high rate of treatment discontinuation [14]. Therefore, to overcome adherence problems, there is a strong need for preventive treatment that is both well tolerated and has an early onset of action [15].

More recently, preventive treatments that target the calcitonin gene-related peptide (CGRP) or the CGRP receptor have been extensively investigated. Among these, monoclonal antibodies that target the CGRP pathway have the advantage of being highly specific in their mechanism of action [14]. The rapidity of onset of monoclonal antibodies against CGRP has been the focus of several studies. In the earliest of these studies, posthoc analyses of two pivotal trials of erenumab for CM $(n=667)$ and EM $(n=955)$ were conducted [16]. Rapid onset of efficacy of erenumab compared with placebo was observed in terms of change from baseline in weekly migraine days (WMD) and achievement of $\geq 50 \%$ reduction in WMD. Furthermore, a fewer erenumab-treated patients experienced migraine on a daily basis compared with placebo during the first week of treatment. Analyses based on two double-blind, randomized, Phase 3 studies comparing galcanezumab with placebo in EM patients (EVOLVE-1, EVOLVE-2) found galcanezumab-treated patients had significantly higher odds of having fewer migraine headache days in the first week and in each subsequent week compared with placebo [17]. Onset of effect was seen as early as the first day after injection $[17,18]$. A post-hoc analysis based on the CONQUER phase $3 \mathrm{~b}$ study of patients with prior treatment failure found that, compared with placebo, galcanezumabtreated patients had a significantly greater reduction in monthly migraine headache days starting at month 1 , and in weekly migraine headache days starting at week 1 with the early onset of effect of galcanezumab considered to begin the day after treatment initiation [19]. Onset of efficacy for fremanezumab in CM was assessed as part of the phase 3 HALO trial [20]. During the 4-week period after the first dose, the mean number of monthly headache days of at least moderate severity was reduced for the all-fremanezumab group compared with the placebo group. Treatment effects were observed at week 1 for the all-fremanezumab group, with separation from placebo by Day 2 (1 day after first injection). Monthly average number of migraine days and the mean number of monthly headache hours showed similar effects.

Two recent phase $2 b / 3$ studies have established the efficacy of fremanezumab in Japanese and Korean patients with EM or CM, respectively $[21,22]$. Among patients with CM $(n=571)$, the least-squares mean ( \pm standard error [SE]) reduction in the average number of headache days of at least moderate severity per month during 12 weeks (primary endpoint) was significantly greater with fremanezumab monthly $(-4.1 \pm 0.4)$ and fremanezumab quarterly $(-4.1 \pm 0.4)$ than with placebo $(-2.4 \pm 0.4)$ [21]. Similarly, among patients with EM $(n=357)$, least-squares mean $( \pm$ SE) reductions in the average number of migraine days per month during 12 weeks (primary endpoint) were significantly greater with fremanezumab monthly $(-4.0 \pm 0.4, n=121)$ and fremanezumab quarterly $(-4.0 \pm 0.4, n=117)$ than with placebo $(-1.0 \pm 0.4, n=116 ; P<0.0001$ for both comparisons) [22]. Improvements in secondary endpoints and a similar rate of adverse events, except injection-site reactions in the case of EM patients, with placebo were also observed with fremanezumab in both studies [21, 22].

Based on this background of previous studies, this subanalysis of two phase $2 b / 3$ studies represents the first study to investigate the onset of efficacy of the CGRP monoclonal antibody, fremanezumab, in East Asian populations.

\section{Methods}

\section{Study design}

This study represents a subanalysis of two multicenter, randomized, double-blind, placebo-controlled, parallel-group trials in Japanese and Korean patients with CM (Clinicaltrials.gov, NCT03303079) and EM (NCT03303092). The study design, populations, inclusion and exclusion criteria have been published in full previously. In brief, the EM trial randomly assigned 357 patients to subcutaneous fremanezumab monthly ( $225 \mathrm{mg}$ at baseline, weeks 4 and 8), fremanezumab quarterly (675 $\mathrm{mg}$ at baseline and placebo at weeks 4 and 8), or matching placebo (Fig. 1). The primary endpoint of the EM trial was the mean change from baseline in the monthly average number of migraine days during the 12 -week treatment period after the first dose. The CM trial randomized 571 patients to subcutaneous fremanezumab monthly $(675 \mathrm{mg}$ at baseline and $225 \mathrm{mg}$ at weeks 4 and 8), fremanezumab quarterly ( $675 \mathrm{mg}$ at baseline and placebo at weeks 4 and 8), or matching placebo (Fig. 1). The primary endpoint of the CM trial was the mean change from baseline in the monthly (28-day) average number of headache days of at least moderate severity during the 12 weeks after the first dose. These studies included prespecified secondary and exploratory analyses and post hoc analyses not previously specified for results up to 1 week.

\section{Study objective and outcomes}

The overall objective of the subanalyses was to evaluate the efficacy of fremanezumab during the initial 4 weeks of treatment in patients with EM or CM. 


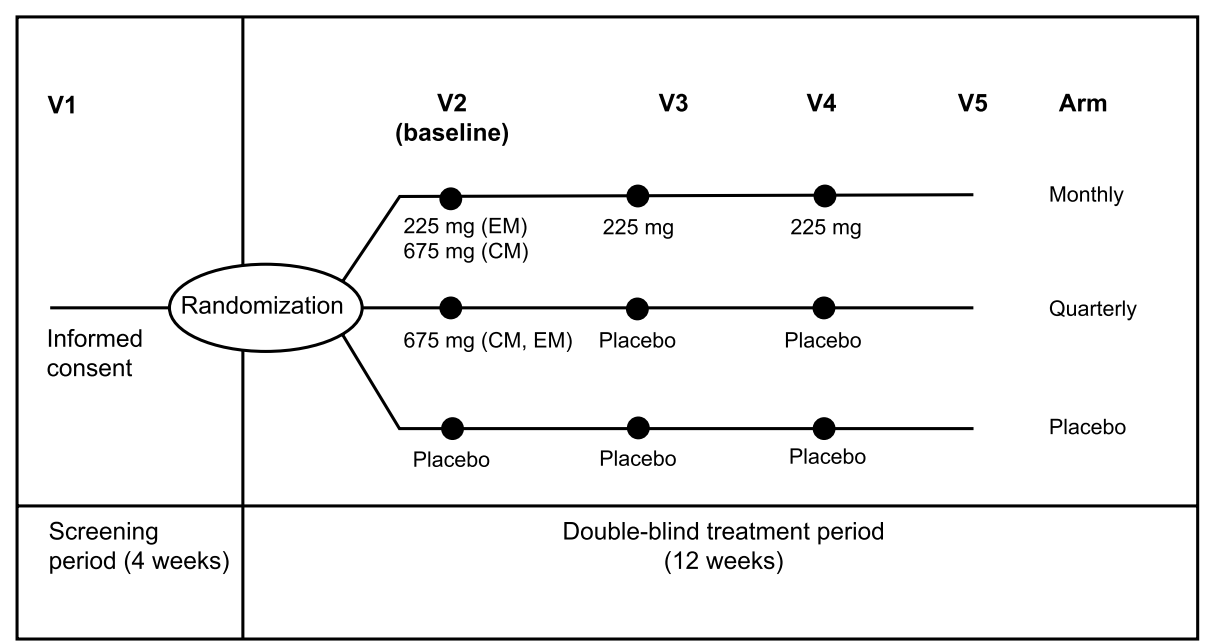

Fig. 1 Study schema in CM and EM patients

In EM patients, the change in monthly migraine days (MMD, days/month) and the change in WMD (days/ week) per week during the first 4 weeks from the first dose were assessed. In addition, EM patients were assessed in terms of the percentage of patients with a migraine day (from day 1 [day of the first injection] to day 7). EM patients were considered in terms of response to fremanezumab quarterly and fremanezumab monthly separately.

In CM patients, the change in headache days of at least moderate severity (days/month) and the change in headache days of at least moderate severity (days/week) per week during the first 4 weeks from the first dose were assessed. Patients with CM were also assessed in terms of the percentage of patients with a headache day of at least moderate severity (from day 1 to day 7). CM patients were considered in terms of response to fremanezumab quarterly and fremanezumab monthly combined.

\section{Statistics}

An ANCOVA model was used to show the mean change in MMD (EM patients) and monthly headache days of at least moderate severity (CM patients) during the 4-week period from the first dose. An MMRM analysis was used to estimate the mean change from baseline in WMD (EM patients) and the change in weekly headache days of at least moderate severity (CM patients) per week during the 4-week period from the first dose. The percentages of patients with a migraine day (from day 1 to day 7) in EM patients and with a headache day of at least moderate severity (from day 1 to day 7) in CM patients were also calculated as part of post hoc analyses. Finally, twosided $95 \%$ confidence intervals $(\mathrm{CI})$ and $P$ values with statistical significance set at $P<0.05$ were constructed for the least-squares mean (LSM) and standard error of the mean (SEM) differences between each fremanezumab group and the placebo group. A headache day of at least moderate severity and a migraine day were normalized to 28 days for the monthly analysis and 7 days for the weekly analysis.

For CM patients, analyses of changes during the first 4 weeks after the first dose compared the placebo group with the "all-fremanezumab" group, which consisted of patients from both the fremanezumab quarterly and monthly groups. In both CM and EM patients, monthly variables with $<10$ days of data and weekly variables with $<3$ days of data were considered missing.

SAS version 9.4 (SAS Institute, Cary, NC) was used for all statistical calculations.

\section{Results}

\section{Subject disposition and baseline characteristics}

In total, 357 patients with EM and 571 patients with CM were included in the EM and CM phase $2 b / 3$ studies. Patient baseline characteristics have been reported previously but are summarized in Table 1.

\section{Efficacy}

Regarding EM patients, both fremanezumab quarterly and fremanezumab monthly led to statistically significant greater reductions from baseline in MMD compared with placebo. Figure 2A shows the mean change in MMD by ANCOVA analysis during the 4-week period after the first dose, at which point the mean (SD) baseline was 9.0 (2.8), 8.6 (2.5) and 8.7 (2.5) days in the placebo, fremanezumab monthly, and fremanezumab quarterly groups, respectively. The LSM (SEM) change from baseline in MMD was equivalent to $-4.41(0.44)$ days for 
Table 1 Patient demographics and clinical characteristics

\begin{tabular}{|c|c|c|c|c|c|}
\hline & \multicolumn{3}{|c|}{ EM, Study NCT03303092 } & \multicolumn{2}{|c|}{ CM, Study NCT03303079 } \\
\hline & \multirow{2}{*}{$\begin{array}{l}\text { Placebo } \\
(n=117)\end{array}$} & \multicolumn{2}{|l|}{ Fremanezumab } & \multirow{2}{*}{$\begin{array}{l}\text { Placebo } \\
(n=191)\end{array}$} & \multirow{2}{*}{$\begin{array}{l}\text { Fremanezumab } \\
\text { Total }(n=380)\end{array}$} \\
\hline & & Monthly $(n=121)$ & Quarterly $(n=119)$ & & \\
\hline Age, years, mean (SD) & $44.2(10.7)$ & $44.4(9.5)$ & $41.9(10.1)$ & $42.1(10.2)$ & $43.1(10.2)$ \\
\hline Body mass index, mean (SD) & $22.8(3.5)$ & $23.0(4.0)$ & $22.5(3.4)$ & $22.8(3.4)$ & $22.9(3.8)$ \\
\hline Female sex, n (\%) & $100(85.5)$ & $101(83.5)$ & $101(84.9)$ & $163(85.3)$ & $328(86.3)$ \\
\hline \multicolumn{6}{|l|}{ Disease history } \\
\hline Time since onset of migraine, years, mean (SD) & $19.4(13.3)$ & $22.0(12.9)$ & $18.3(11.4)$ & $19.0(11.2)$ & $18.5(12.3)$ \\
\hline \multirow[t]{2}{*}{ Use of preventive migraine medication at baseline, yes, n (\%) } & $22(18.8)$ & $24(19.8)$ & $23(19.3)$ & $41(21.5)$ & $79(20.8)$ \\
\hline & $n=117$ & $n=121$ & $n=118$ & $n=191$ & $n=378$ \\
\hline \multicolumn{6}{|l|}{ Disease characteristics during 28-day pretreatment period } \\
\hline $\begin{array}{l}\text { Number of days with headache of any severity and dura- } \\
\text { tion, mean (SD) }\end{array}$ & $11.1(2.5)$ & $11.0(2.1)$ & $11.0(2.5)$ & $21.2(4.3)$ & $21.4(4.0)$ \\
\hline $\begin{array}{l}\text { Number of headache days of at least moderate severity, } \\
\text { mean (SD) }\end{array}$ & $8.0(2.8)$ & $7.6(2.5)$ & $7.5(2.8)$ & $13.5(5.0)$ & $13.3(5.4)$ \\
\hline Number of migraine days, mean (SD) & $9.0(2.8)$ & $8.6(2.5)$ & $8.7(2.5)$ & $15.4(5.0)$ & $15.8(5.2)$ \\
\hline Use of any acute headache medications, yes, n (\%) & $117(100.0)$ & $120(99.2)$ & $117(98.3)$ & $191(100.0)$ & $375(98.7)$ \\
\hline $\begin{array}{l}\text { Use of migraine-specific acute headache medications, yes, } \\
\text { (\%) }\end{array}$ & $114(97.4)$ & $115(95.0)$ & $110(92.4)$ & $177(92.7)$ & $352(92.6)$ \\
\hline
\end{tabular}

SD Standard deviation

fremanezumab quarterly, $-3.63(0.43)$ days for fremanezumab monthly, and $-0.50(0.44)$ days for placebo. This equated to a difference in LSM change from baseline of -3.91 (95\% CI $-4.80,-3.02 ; P<0.0001)$ days/month for fremanezumab quarterly versus placebo and -3.13 (95\% CI $-4.01,-2.24 ; P<0.0001)$ days/month for fremanezumab monthly versus placebo. Figure $2 \mathrm{~B}$ shows the mean change in WMD by MMRM analysis per week during the 4-week period after the first dose, at which the mean (SD) baseline was 2.2 (0.7), $2.2(0.6)$, and $2.2(0.6)$ days in the placebo, fremanezumab monthly, and fremanezumab quarterly groups, respectively. These also showed statistically significant differences for both fremanezumab quarterly and fremanezumab monthly versus placebo.

Similarly, in CM patients, fremanezumab led to a greater reduction from baseline in headache days of at least moderate severity (days/month) compared with placebo. Figure 3A shows the mean change in monthly headache days of at least moderate severity by ANCOVA analysis during the 4-week period from the first dose, at which the mean (SD) baseline was 13.5 (5.0), and 13.3 (5.4) days in the placebo and fremanezumab groups, respectively. Specifically, the fremanezumab group had an LSM (SEM) change from baseline of $-4.06(0.41)$ days/month compared with -1.56 (0.47) days/month for placebo. This equated to a difference in LSM change from baseline of $-2.51(95 \% \mathrm{CI}-3.33,-1.68 ; P<0.0001)$ days/ month for fremanezumab versus placebo. Reductions in the number of headache days of at least moderate severity during the first 4 weeks showed statistically significant differences for fremanezumab versus placebo at each time point. Figure $3 \mathrm{~B}$ shows the mean change in headache days of at least moderate severity by MMRM analysis per week during the 4-week period from the first dose, at which the mean (SD) baseline was 3.4 (1.2), and 3.3 (1.3) days in the placebo, and fremanezumab groups, respectively.

Figure 4 and 5 show the percentage of patients with a migraine day and the percentage of patients with a headache day at least moderate severity from Day 1 (day of first injection) to Day 7 in EM patients and CM patients, respectively. In both populations, the percentage of patients was noticeably lower in fremanezumab-treated patients from as early as Day 2 (the day after the first injection) through to Day 7. In EM patients who received fremanezumab quarterly, the percentage of patients with a migraine day was lower as early as Day 1 (day of first injection).

\section{Discussion}

Early onset of effect is rated as a highly important feature of migraine preventive treatment by patients and has been linked to improvements in adherence [23]. A previous post hoc analysis of the phase 3 HALO trial examining early onset found greater early reductions in the mean number of monthly headache days of at least moderate severity, monthly average number of migraine 

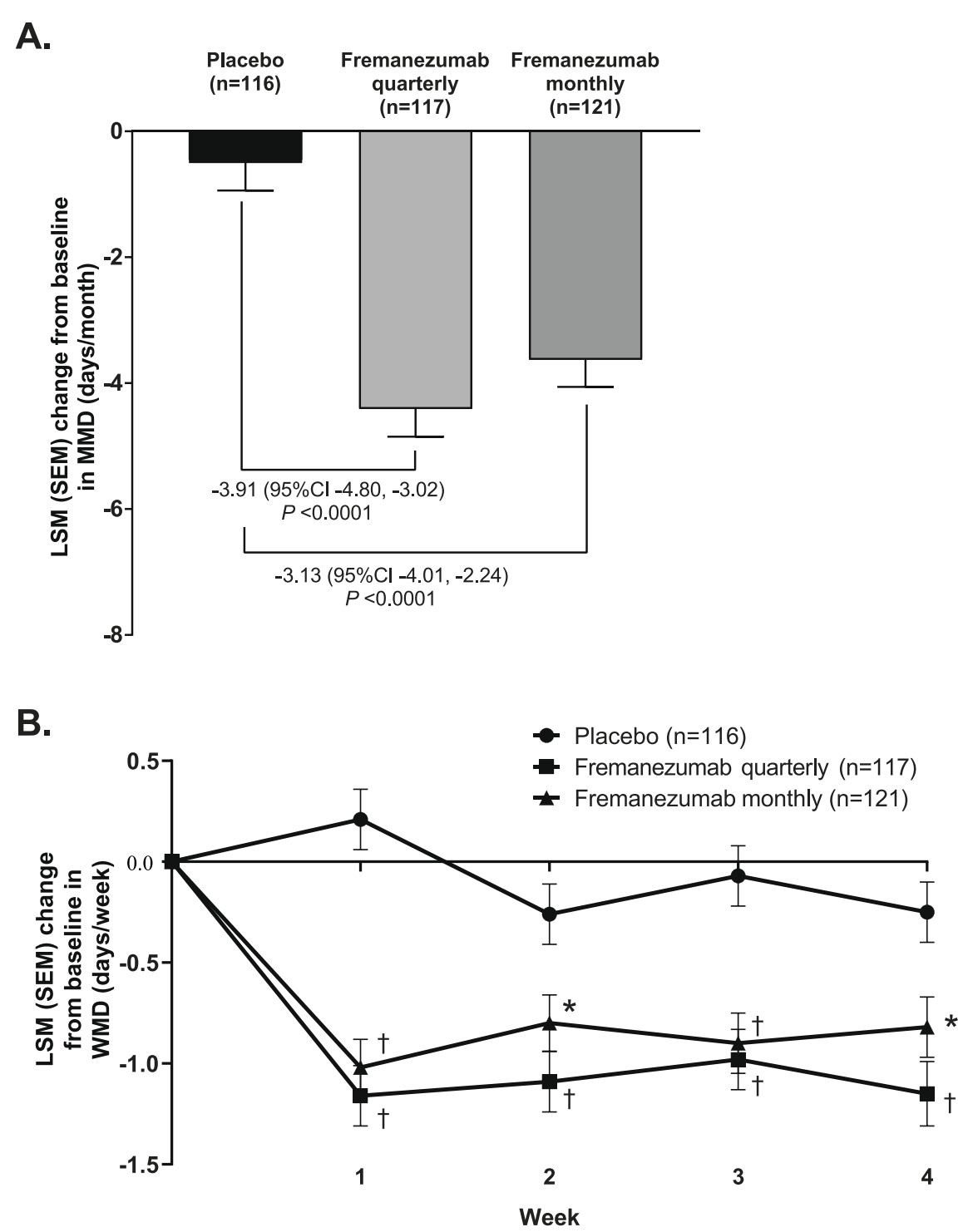

Fig. 2 Change in (A) MMD and (B) WMD in EM patients. Change in MMD represents mean change from baseline during the 4-week period from the first dose (ANCOVA analysis) while change in WMD represents mean change per week during the 4-week period from the first dose (MMRM analysis). An asterisk denotes $P<0.05$ and a dagger $P<0.0001$ for the comparison with placebo. Abbreviations: ANCOVA, analysis of covariance; LSM, least-squares mean; MMD, monthly migraine days; MMRM, mixed-effects model for repeated measures; WMD, weekly migraine days

days and mean number of monthly headache hours for CM patients treated with fremanezumab versus placebo [20]. These subanalyses of early onset similarly found that preventive treatment with fremanezumab reduced MMD in EM patients and headache days of at least moderate severity in CM patients. Differences in efficacy endpoints between fremanezumab and placebo were sustained throughout the 4-week treatment period in these analyses. Further, the differential effect of fremanezumab versus placebo became evident during the first week of treatment and was even observed as early as the first day of injection in EM patients and the day after first injection in CM patients. These results suggest that fremanezumab has an early onset of action with clinical benefits for patients, with potential impact on patient satisfaction and adherence.

Two previous Phase $2 \mathrm{~b} / 3$ studies in Japanese and Korean patients, which formed the basis of these subanalyses, established the efficacy of fremanezumab quarterly and monthly for periods up to 12 weeks [21, 22]. According to the primary endpoints, the reduction in the average number of migraine days (EM) or headache 
A.

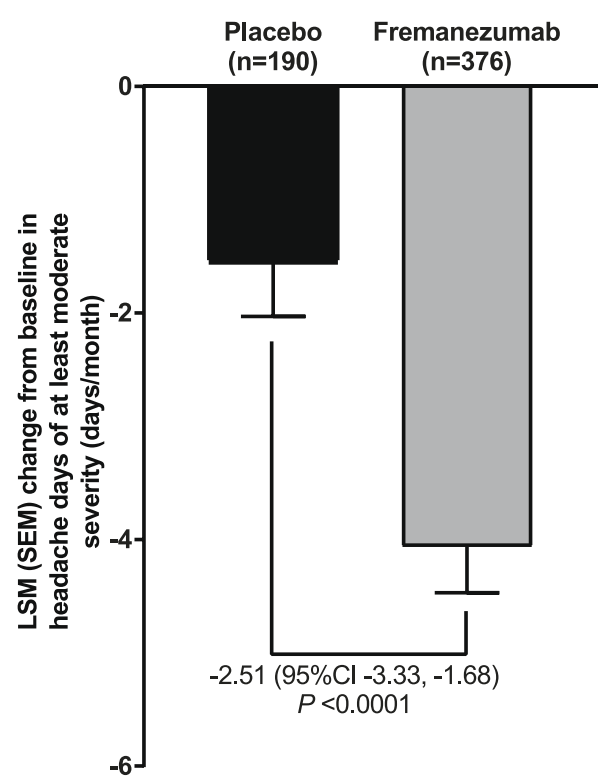

B.

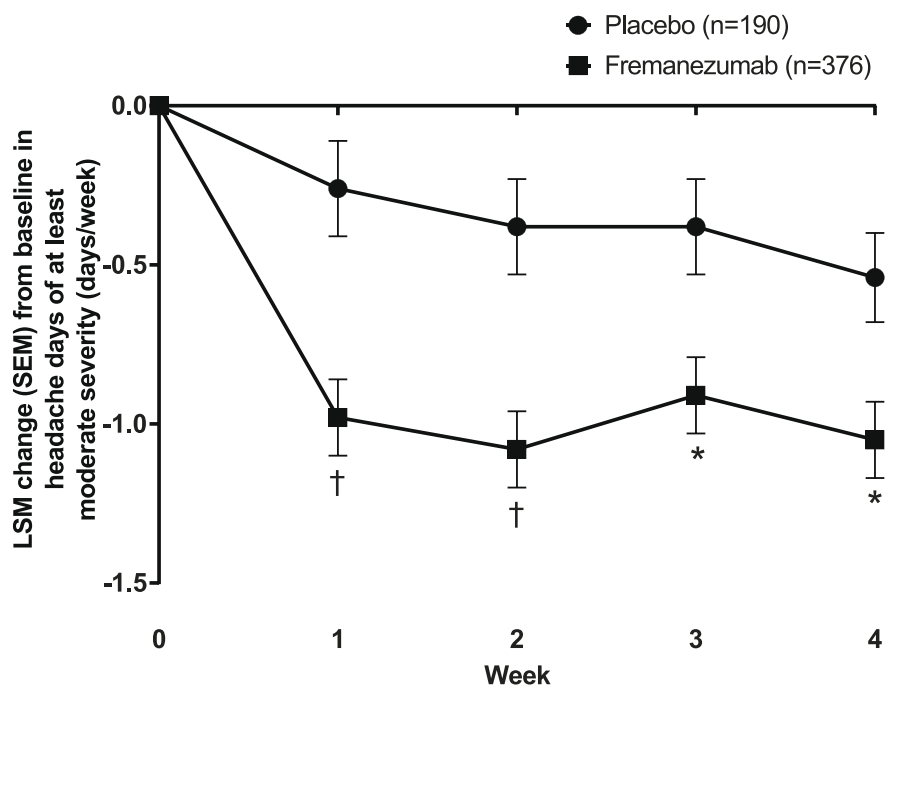

Fig. 3 Change in (A) monthly and (B) weekly average headache days of at least moderate severity in CM patients. Change in monthly average number of headache days of at least moderate severity represents mean change from baseline during the 4-week period after the first dose (ANCOVA analysis) while change in the weekly average number of headache days of at least moderate severity represents mean change per week during the 4-week period from the first dose (MMRM analysis). An asterisk denotes $P<0.05$ and a dagger $P<0.0001$ for the comparison with placebo. Fremanezumab is the sum of fremanezumab monthly and fremanezumab quarterly groups. Abbreviations: ANCOVA, analysis of covariance; LSM, least-squares mean; MMRM, mixed-effects model for repeated measures

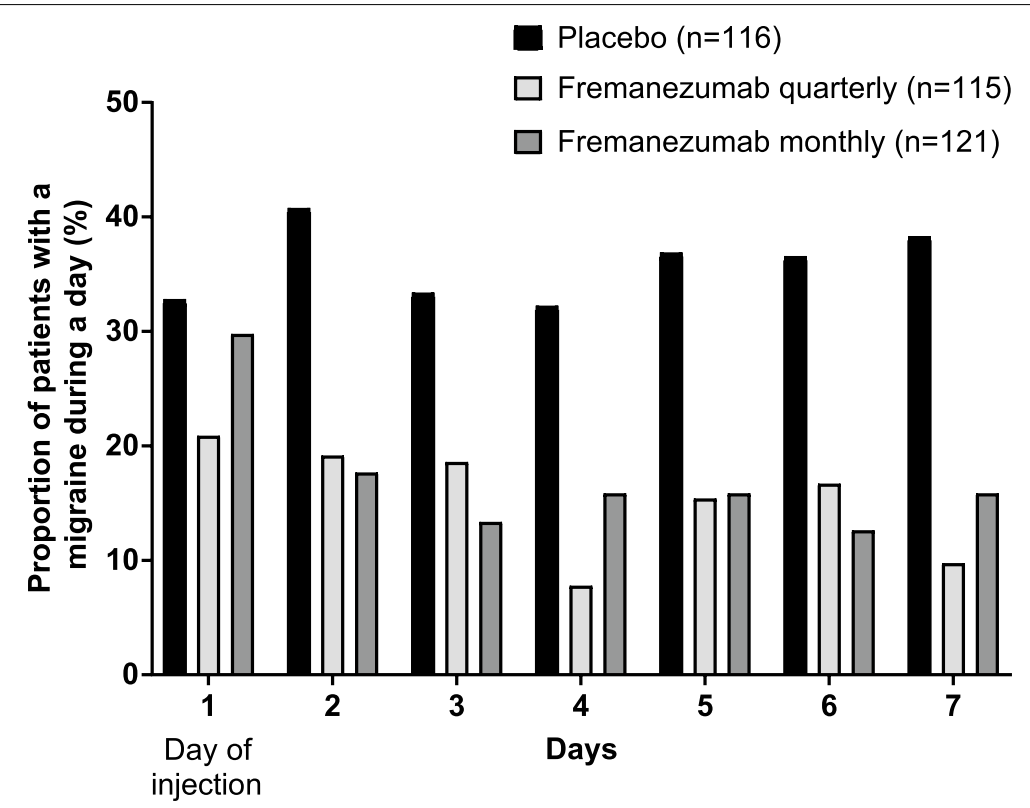

Fig. 4 Percentage of EM patients reporting a migraine during a day from Day 1 to 7 . Day 1 is the day of injection of study medications. $P<0.05$ for difference with placebo from Day 1-7 for fremanezumab quarterly and from Day 2-7 for fremanezumab monthly 


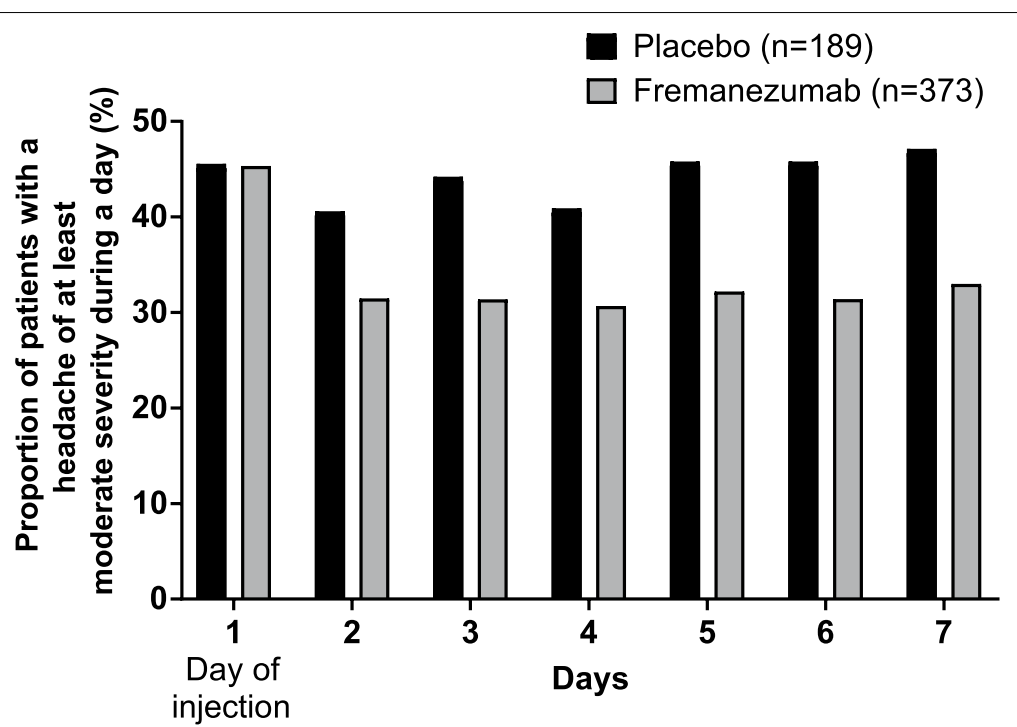

Fig. 5 Percentage of CM patients reporting a headache during a day from Day 1 to 7 . Headache in CM patients was defined as those of at least moderate severity. Day 1 is the day of injection of study medications. Fremanezumab is the sum of fremanezumab monthly and fremanezumab quarterly groups. $P<0.05$ for difference with placebo from Day $2-7$ for fremanezumab

days of at least moderate severity $(\mathrm{CM})$ per month during 12 weeks was significantly greater with fremanezumab monthly and fremanezumab quarterly than with placebo. Further, improvements in all secondary endpoints were also noted in both studies. Over long-term observation, both fremanezumab monthly and quarterly reduced the monthly number of migraine days in a 52-week, randomized, double-blind extension of the HALO trials [24]. A subanalysis of these long-term HALO results similarly found that fremanezumab monthly and quarterly led to sustained improvements in monthly migraine days and headache days of at least moderate severity throughout 12 months in Japanese patients with CM and EM.

Without minimizing the importance of long-term efficacy, there is also a clear patient preference for early efficacy benefits for migraine prevention [23], which has been a limitation of existing oral preventive medications. Indeed, in a patient survey, speed of onset was rated as the second most important aspect of preventive treatment besides actual effectiveness [23]. Switching between oral agents is a common practice to optimize therapy but persistence appears to actually worsen as patients cycle through agents [25].

Previously, early onset of efficacy has been shown with fremanezumab in CM patients [20], EM patients [26] and $\mathrm{CM}$ or EM patients with inadequate response to 2-4 prior migraine preventive treatment classes, including $\geq 1$ inadequate response due to lack of efficacy [27, 28], erenumab in EM and CM patients [16] and galcanezumab in EM patients enrolled in the EVOLVE-1 and EVOLVE-2 studies [17], as well as in EM or CM patients with previous preventive medication failure [19]. Results from the present subgroup analysis were consistent with these previous studies of fremanezumab and other anti-CGRP pathway monoclonal antibodies. In the previous post hoc analysis during the first 4 weeks of a primary trial of fremanezumab in CM patients, the statistically significant difference in LSM (95\% CI) change in headache days of at least moderate severity per month between fremanezumab total and placebo $(-2.3[-3.0,-1.7], P<0.0001)$ was highly similar to the difference in $\mathrm{CM}$ patients in this analysis $(-2.51[-3.33,-1.68], P<0.0001)$ [20]. In EM patients, corresponding differences in MMD between fremanezumab and placebo in this analysis were also significant for both fremanezumab monthly $(-3.13$ $[-4.01,-2.24], P<0.0001)$ and fremanezumab quarterly $(-3.91[-4.80,-3.02], P<0.0001)$. Post hoc analyses of the FOCUS study in CM or EM patients with inadequate response to 2-4 prior migraine preventive treatment classes, including $\geq 1$ inadequate response due to lack of efficacy also showed consistent results [27, 28]. In these analyses, the LSM (SE) change from baseline in the monthly average number of migraine days over the first 4 weeks of treatment was -4.1 for both fremanezumab quarterly and fremanezumab monthly (versus -0.6 for placebo, $P<0.0001$ for both comparisons) [28]. Onset of action was also rapid with a significant reduction in the odds of experiencing a migraine with fremanezumab versus placebo noted from Day 2 (1 day after first injection) to Day 7 [27].

Significant differences between active treatment and placebo were also observed at 4 weeks in studies of other 
anti-CGRP pathway monoclonal antibodies $[16,17,19]$. Further analyses for each week up to 4 weeks found significant reductions in WMD or headache days of at least moderate severity per week in EM and CM patients, respectively, from week 1 in the present analysis, which continued through to week 4 . Onset of effect at week 1 was also identified in the previous post hoc analysis of fremanezumab [20], as well as in studies of erenumab and galcanezumab [16, 17, 19]. Finally, additional analyses of efficacy within the first week were conducted in the present and previous analyses. In the present subanalysis, the percentage of patients with either a migraine day (EM patients) or headache day at least moderate severity (CM patients) was noticeably lower in fremanezumab-treated patients from as early as the day of the first injection in EM patients treated with fremanezumab quarterly or the day after the first injection in CM patients and EM patients treated with fremanezumab monthly. This, as previously noted, is consistent with the results for fremanezumab in post hoc analyses of the FOCUS study [27]. In the post hoc analysis of the phase 3b CONQUER study, onset of effect for galcanezumab was determined to occur the day following the first injection in EM or CM patients with previous preventive medication failures [19]. Similarly, in the analysis of the EVOLVE-1 and EVOLVE-2 studies in EM patients, the estimated proportion of patients experiencing migraine was significantly lower with galcanezumab compared with placebo from the day after the first injection [17]. Results for erenumab varied from day 3 to day 7 after injection depending on the dose and patient population [16].

Taken together, these results suggest that anti-CGRP pathway monoclonal antibodies, including fremanezumab, can provide early onset of action and thereby reduce the potential for patients to discontinue treatment. Discontinuation is a recognized disadvantage with oral preventive medications, which require daily adherence and titration to effect as well as potentially leading to headache chronification and medication overuse headaches through overuse of acute medications and tolerance $[11,14,29]$. Subanalyses of trials of antiCGRP pathway monoclonal antibodies, including fremanezumab, have shown the potential of these agents to reduce the use of acute migraine treatment and lower the potential for medication overuse headaches [30, 31].

One of the main limitations of this subanalysis is that only Japanese and Korean patients were included. Nevertheless, the results are consistent with similar analysis from global study population. Further, this study is not powered to confirm treatment differences from placebo at early time points. Despite this limitation, substantial treatment differences were still demonstrated. Finally, assessment of migraine days and headache days of at least moderate severity were based on headache diary and therefore the influence of subjectivity in individual patient assessment cannot be ruled out.

In conclusion, fremanezumab shows a rapid onset of action in reducing migraine days and headache days at least moderate severity in EM patients and CM patients, respectively. Treatment effects were noted as early as the first day of injection in EM patients treated with fremanezumab quarterly and as early as the day after first injection in other patients. These results in Japanese and Korean patients align with those noted in subanalyses of fremanezumab and other anti-CGRP pathway monoclonal antibodies. In addition to the long-term efficacy and favorable safety and tolerability profile, the lack of need for titration, and flexible monthly or quarterly dosing schedule, the early onset of fremanezumab provides the potential to reduce barriers to adherence.

\begin{abstract}
Abbreviations
ANCOVA: Analysis of covariance; CGRP: Calcitonin gene-related peptide; Cl: Confidence interval; CM: Chronic migraine; EM: Episodic migraine; LSM: Leastsquares mean; MMD: Monthly migraine days; MMRM: Mixed-effects model for repeated measures; SD: Standard deviation; SE: Standard error; SEM: Standard error of the mean; WMD: Weekly migraine days.
\end{abstract}

\section{Acknowledgments}

The authors thank all participants and clinicians for their involvement and contribution to the study. The authors also thank Yoshiko Okamoto, PhD, and Mark Snape, MBBS, of inScience Communications, Springer Healthcare, for helping write the outline and first draft of the manuscript. This medical writing assistance was funded by Otsuka Pharmaceutical Co., Ltd.

\section{Authors' contributions}

$T T, M N, Y S, M I$ and NK were involved in study concept and design. TT, BK, MN, YS, MI and NK were involved in acquisition of data. All authors were involved in analysis and interpretation of data, drafting of the manuscript, and final approval of the completed manuscript.

\section{Funding}

This study was funded by Otsuka Pharmaceutical Co., Ltd.

\section{Availability of data and materials}

The datasets analyzed during the current study are not publicly available but anonymized individual participant data are available from the corresponding author on reasonable request to achieve aims pre-specified in a methodologically sound research proposal.

\section{Declarations}

\section{Ethics approval and consent to participate}

Both trials were conducted in compliance with the International Conference on Harmonisation Good Clinical Practice Guideline and local regulatory requirements, including those related to informed consent, and were approved by an institutional review board or independent ethics committee/ ethics committee.

\section{Consent for publication}

Not applicable.

\section{Competing interests}

TT has received grants or contracts from Eisai Co., Ltd. Amgen Inc., Eli Lilly Japan K.K., Allergan Japan K.K.; consulting fees from Otsuka Pharmaceutical Co., Ltd.; honoraria for lectures from Otsuka Pharmaceutical Co., Ltd., Eisai Co., 
Ltd., Amgen Inc., and Eli Lilly Japan K.K. B-KK reports personal fees from Otsuka Pharmaceutical Co., Ltd.; consultation fees from Teva Korea and Sanofi Korea; consultation and lecture fees from Lundbeck Korea; and lecture fees from Lilly Korea, Allergan Korea, SK-Pharma, and YuYu Pharma Inc. XN is a full-time employee of Teva Branded Pharmaceutical Products R\&D. MN, YS, MI, and NK are full-time employees of Otsuka Pharmaceutical Co., Ltd.

\section{Author details}

${ }^{1}$ Headache Center, Department of Neurology, Tominaga Hospital, 1-4-48 Minatomachi Naniwa-ku, Osaka-shi, Osaka 556-0017, Japan. ${ }^{2}$ Medical Affairs, Otsuka Pharmaceutical Co., Ltd., 3-2-27 Otedori, Chuo-ku, Osaka 540-0021, Japan. ${ }^{3}$ Medical Affairs, Otsuka Pharmaceutical Co., Ltd., Shinagawa Grand Central Tower, 2-16-4 Konan, Minato-ku, Tokyo 108-8242, Japan. ${ }^{4}$ Headquarters of Clinical Development, Otsuka Pharmaceutical Co., Ltd., 3-2-27 Otedori, Chuo-ku, Osaka 540-0021, Japan. ${ }^{5}$ Nowon Eulji Medical Center, Eulji University School of Medicine, 68 Hangeulbiseok-ro, Nowon-gu, Seoul 01830, Republic of Korea. ${ }^{6}$ Speciality Clinical Development, Teva Branded Pharmaceutical Products R\&D, Inc., 145 Brandywine Pkwy, West Chester, PA 19380, USA. ${ }^{7}$ Medical Affairs, Otsuka Pharmaceutical Co., Ltd., 463-10 Kagasuno, Kawauchi-cho, Tokushima 771-0192, Japan.

\section{Received: 5 December 2021 Accepted: 21 January 2022}

Published online: 09 February 2022

\section{References}

1. GBD (2017) Disease and injury incidence and prevalence collaborators (2018) global, regional, and national incidence, prevalence, and years lived with disability for 354 diseases and injuries for 195 countries and territories, 1990-2017: a systematic analysis for the global burden of disease study 2017. Lancet 392(10159):1789-1858

2. Buse DC, Scher Al, Dodick DW, Reed ML, Fanning KM, Manack Adams A et al (2016) Impact of migraine on the family: perspectives of people with migraine and their spouse/domestic partner in the CaMEO study. Mayo Clin Proc S0025-6196(16):00126-00129

3. D’Amico D, Grazzi L, Usai S, Leonardi M, Raggi A (2013) Disability and quality of life in headache: where we are now and where we are heading. Neurol Sci 34(Suppl 1):S1-S5

4. Ferracini GN, Dach F, Speciali JG (2014) Quality of life and health-related disability in children with migraine. Headache 54(2):325-334

5. Lipton RB, Bigal ME, Kolodner K, Stewart WF, Liberman JN, Steiner TJ (2003) The family impact of migraine: population-based studies in the USA and UK. Cephalalgia 23(6):429-440

6. Pradeep R, Nemichandra SC, Harsha S, Radhika K (2020) Migraine disability, quality of life, and its predictors. Ann Neurosci 27(1):18-23

7. Bonafede M, Wilson K, Xue F (2019) Long-term treatment patterns of prophylactic and acute migraine medications and incidence of opioid-related adverse events in patients with migraine. Cephalalgia 39(9):1086-1098

8. Hepp Z, Dodick DW, Varon SF, Gillard P, Hansen RN, Devine EB (2015) Adherence to oral migraine-preventive medications among patients with chronic migraine. Cephalalgia 35(6):478-488

9. Woolley JM, Bonafede MM, Maiese BA, Lenz RA (2017) Migraine prophylaxis and acute treatment patterns among commercially insured patients in the United States. Headache 57(9):1399-1408

10. Blumenfeld AM, Bloudek LM, Becker WJ, Buse DC, Varon SF, Maglinte GA et al (2013) Patterns of use and reasons for discontinuation of prophylactic medications for episodic migraine and chronic migraine: results from the second international burden of migraine study (IBMS-II). Headache 53(4):644-655

11. Loder EW, Rizzoli P (2011) Tolerance and loss of beneficial effect during migraine prophylaxis: clinical considerations. Headache 51(8):1336-1345

12. Ueda K, Ye W, Lombard L, Kuga A, Kim Y, Cotton S et al (2019) Real-world treatment patterns and patient-reported outcomes in episodic and chronic migraine in Japan: analysis of data from the Adelphi migraine disease specific programme. J Headache Pain 20(1):68

13. Loder E, Rizzoli P (2018) Pharmacologic prevention of migraine: a narrative review of the state of the art in 2018. Headache 58(Suppl 3):218-229

14. Vikelis M, Spingos KC, Rapoport AM (2018) A new era in headache treatment. Neurol Sci 39(Suppl 1):47-58
15. D'Amico D, Tepper SJ (2008) Prophylaxis of migraine: general principles and patient acceptance. Neuropsychiatr Dis Treat 4(6):1155-1167

16. Schwedt T, Reuter U, Tepper S, Ashina M, Kudrow D, Broessner G et al (2018) Early onset of efficacy with erenumab in patients with episodic and chronic migraine. J Headache Pain 19(1):92

17. Detke HC, Millen BA, Zhang Q, Samaan K, Ailani J, Dodick DW et al (2020) Rapid onset of effect of galcanezumab for the prevention of episodic migraine: analysis of the EVOLVE studies. Headache 60(2):348-359

18. Andreou AP, Wright P, Detke H, Ruff D, Reuter U (2020) Galcanezumab shows efficacy as early as day 1 after initial treatment vs. placebo for the prevention of episodic and chronic migraine (4014). Neurology 94(15 Supplement):4014

19. Schwedt TJ, Kuruppu DK, Dong Y, Standley K, Yunes-Medina L, Pearlman E (2021) Early onset of effect following galcanezumab treatment in patients with previous preventive medication failures. J Headache Pain 22(1):15

20. Winner PK, Spierings ELH, Yeung PP, Aycardi E, Blankenbiller T, GrozinskiWolff M et al (2019) Early onset of efficacy with fremanezumab for the preventive treatment of chronic migraine. Headache 59(10):1743-1752

21. Sakai F, Suzuki N, Kim BK, Igarashi H, Hirata K, Takeshima T et al (2021) Efficacy and safety of fremanezumab for chronic migraine prevention: multicenter, randomized, double-blind, placebo-controlled, parallelgroup trial in Japanese and Korean patients. Headache 61 (7):1092-1101

22. Sakai F, Suzuki N, Kim BK, Tatsuoka Y, Imai N, Ning X et al (2021) Efficacy and safety of fremanezumab for episodic migraine prevention: multicenter, randomized, double-blind, placebo-controlled, parallel-group trial in Japanese and Korean patients. Headache 61(7):1102-1111

23. Peres MF, Silberstein S, Moreira F, Corchs F, Vieira DS, Abraham N et al (2007) Patients' preference for migraine preventive therapy. Headache 47(4):540-545

24. Goadsby PJ, Silberstein SD, Yeung PP, Cohen JM, Ning X, Yang R et al (2020) Long-term safety, tolerability, and efficacy of fremanezumab in migraine. A randomized study. Neurology 95(18):e2487-e2499

25. Hepp Z, Dodick DW, Varon SF, Chia J, Matthew N, Gillard P et al (2017) Persistence and switching patterns of oral migraine prophylactic medications among patients with chronic migraine: a retrospective claims analysis. Cephalalgia 37(5):470-485

26. Brandes J, Yeung PP, Aycardi E, Bigal M, Blankenbiller T, Grozinski-Wolff $\mathrm{M}$ et al (2018) Early onset of action with fremanezumab versus placebo for the preventive treatment of chronic migraine. 60th Annual Scientific Meeting American Headache Society ${ }^{\circledR}$ June 28-July 1, 2018 San Francisco Marriott Marquis San Francisco, CA. Headache 58(S2):61-215

27. Brandes JL, Ramirez Campos V, Yang R, Cohen JM, Galic M, Ning X et al. 2020 Very early onset of action of fremanezumab in patients with migraine and documented inadequate response to 2 to 4 classes of migraine preventive medications: results of the international, multicenter, randomized, placebo-controlled FOCUS study. Presented at the European Headache Federation (EHF) Virtual Congress

28. Starling AJ, Ramirez Campos V, Yang R, Cohen JM, Galic M, Ning X et al. 2020 Early efficacy of fremanezumab in patients with episodic and chronic migraine and inadequate response to 2 to 4 classes of migraine preventive medications due to lack of efficacy: Results of the international, multicenter, phase 3b FOCUS Study. Presented at the European Headache Federation (EHF) Virtual Congress

29. Diener HC, Holle D, Dresler T, Gaul C (2018) Chronic headache due to overuse of analgesics and anti-migraine agents. Dtsch Arztebl Int 115(22):365-370

30. Silberstein SD, Cohen JM, Yang R, Gandhi SK, Du E, Jann AE et al (2021) Treatment benefit among migraine patients taking fremanezumab: results from a post hoc responder analysis of two placebo-controlled trials. J Headache Pain 22(1):2

31. Tepper SJ, Diener HC, Ashina M, Brandes JL, Friedman DI, Reuter U et al (2019) Erenumab in chronic migraine with medication overuse: subgroup analysis of a randomized trial. Neurology 92(20):e2309-e2320

\section{Publisher's Note}

Springer Nature remains neutral with regard to jurisdictional claims in published maps and institutional affiliations. 